AperTO - Archivio Istituzionale Open Access dell'Università di Torino

\title{
Adopting the Mediterranean Diet Score in a Diet Management System
}

\section{This is the author's manuscript}

Original Citation:

\section{Availability:}

This version is available http://hdl.handle.net/2318/1747532

since 2020-07-31T13:04:20Z

Publisher:

SCITEPress

Published version:

DOI:10.5220/0009162506700676

Terms of use:

Open Access

Anyone can freely access the full text of works made available as "Open Access". Works made available under a Creative Commons license can be used according to the terms and conditions of said license. Use of all other works requires consent of the right holder (author or publisher) if not exempted from copyright protection by the applicable law. 


\title{
Adopting the Mediterranean Diet Score in a Diet Management System
}

\author{
Luca Anselma, Mirko Di Lascio, Antonio Lieto and Alessandro Mazzei \\ Dipartimento di Informatica, Università di Torino, Corso Svizzera 185, Torino, Italy \\ \{anselma,lieto,mazzei\}@di.unito.it,mirko.dilascio@edu.unito.it
}

Keywords: Diet Management, Mediterranean Diet, Artificial Intelligence, Constraints, Ontology.

\begin{abstract}
In this work we want to study the possibility to integrate a Mediterranean Diet Score into an existing diet management architecture. The main goal is to integrate the quantitative constraints on macronutrients with the qualitative constraints encoded in the Mediterranean Diet. This paper presents some preliminary results on this roadmap.
\end{abstract}

\section{INTRODUCTION}

Diet formalization can be understood by humans as the fulfillment of some numeric constraints on quantity and time for food consumption. However, for psychological and cognitive reasons, it can be very difficult for a user to understand the numeric constraints regarding micro and macro nutrients. For this specific task, computers can help people both in (1) computing the values of the macronutrients in the meal actually eaten, and (2) reminding the best choices for next meals (Anselma and Mazzei, 2015). However, it remains open the problem of explanation, that is, in order to guide an user to improve its dietetic behaviour, the computer needs to explain in details of the diet transgression (Anselma and Mazzei, 2017).

In contrast to numeric constraints, Mediterranean diet is an example that, at a high level, can be formalized as a set of qualitative constraints on food (Panagiotakos et al., 2007; Panagiotakos et al., 2006). In particular, by assuming a standard quantity for a single portion of an ingredient in a recipe (Società Italiana di Nutrizione Umana (SINU), 2014), we can explain the diet using simple sentences in natural language, for instance Use daily olive oil in cooking. In this way, also diet transgressions and their possible compensation can be communicated to the user in a more transparent and efficient way, e.g. You shouldn't eat again red meat now because this week you have eaten it twice. However, it is not trivial to "integrate" high-level rules of the Mediterranean diet with the food actually eaten for various reasons. For example, if a user eats "caponata", it is not trivial to infer that (i) the dish contains eggplants, (ii) eggplants are vegetables (thus the dish contributes to the con- straint Eat more than 18 servings of vegetables each month, see Table 1), (iii) it is cooked using olive oil (thus, it contributes also to the constraint Use daily olive oil in cooking). Notice that using the recipe of caponata could be not enough. For example, point (ii) is a piece of information not usually available in a recipe (Mazzei, 2014).

In this paper we describe the first steps towards the integration of Mediterranean diet into an exiting framework for the management of the diet called MADiMan (Multimedia Architecture for Diet Management) (Anselma et al., 2018). In previous work, some questions concerning the general architecture of the MADiMan system (Mazzei et al., 2015; Anselma and Mazzei, 2015), concerning the capacities of the reasoning module with a number of computer simulations with virtual agents based on hospital menus (Anselma et al., 2017) and on Mediterranean menus (Anselma et al., 2018) and concerning the Natural Language Generation module (Anselma and Mazzei, 2018) have been addressed.

To the best of our knowledge there is no other proposal for the automatic computation of a Mediterranean score. However, some researchers proposed some forms of perfect diet and implemented systems for their computation. Some authors applied Operational Research techniques for the planning of a perfect diet (see the survey in (Lancaster, 1992) or, more recently, (Bas, 2014)). Operational Research techniques (often based on the simplex method) plan an entire diet and they do not support the user in choosing a meal. In (Buisson, 2008) a user can assess the compatibility of a single meal to a norm and the system suggests some actions for balancing the user's meal. A different approach was proposed in (Mai- 


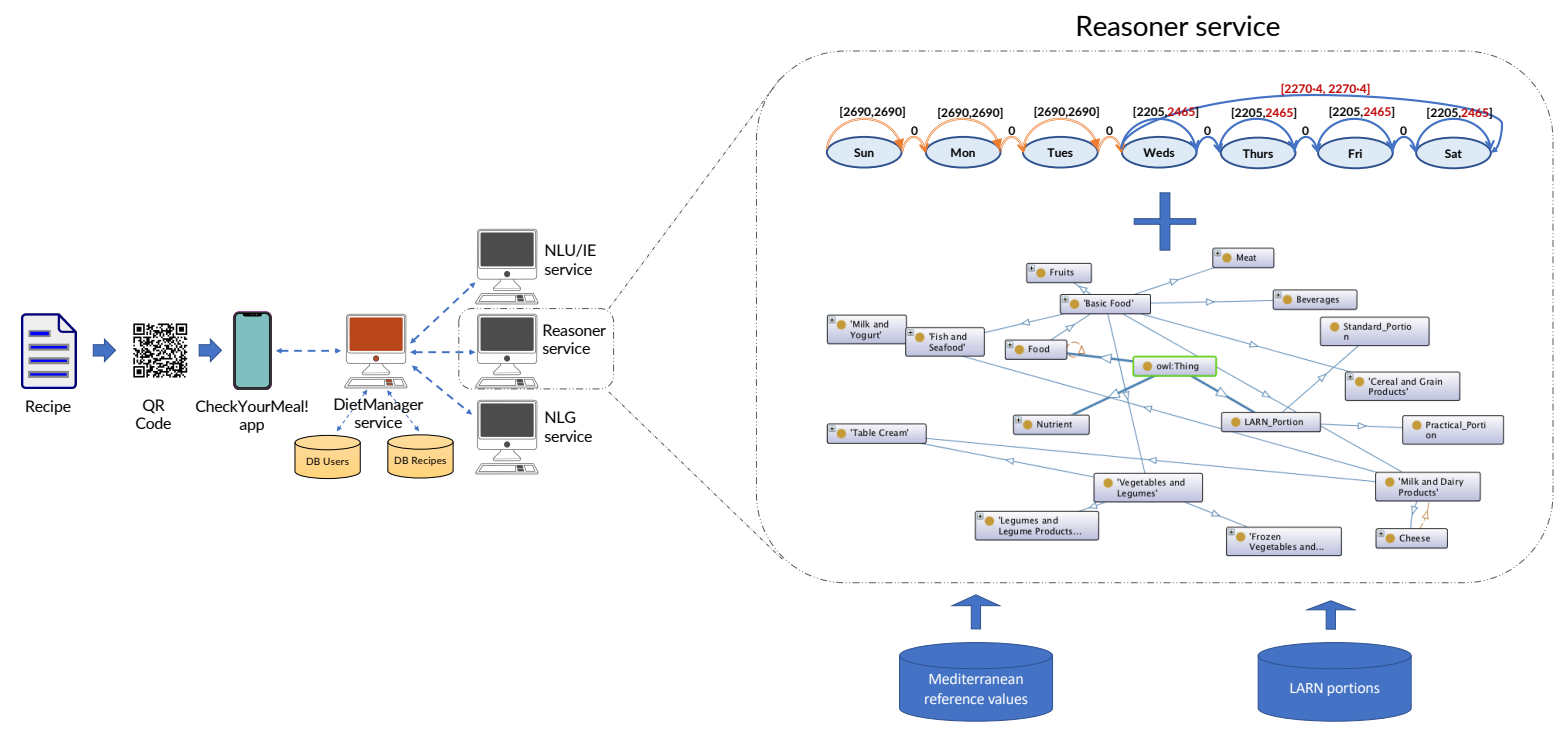

Figure 1: The current MADiMan architecture (on the left) and a detail of the reasoner module (on the right). Note that the reasoning is based on STP numeric constraints for macronutrients, on ontological constraints for Mediterranean References Values (MRVs), and on standard LARN portions definition.

mone et al., 2018), where "a motivational platform for supporting the monitoring of users' behaviors and for persuading them to follow healthy lifestyles" it has been presented. In (Maimone et al., 2018), the authors provide a case study based on diet and physical activity that implements an ontological reasoner.

The specific research questions that we want to investigate on in this paper are (1) the use of ontologies for formalizing the Mediterranean score as defined in (Panagiotakos et al., 2007; Panagiotakos et al., 2006) (see Section 3), (2) the design of a procedure, based on ontological formalization, for the computation of this Mediterranean score on some human-produced data (see Section 4).

\section{THE MADiMan FRAMEWORK}

MADiMan (Multimedia Application for Diet Management) is an ongoing project ${ }^{1}$ with the aim of building a virtual assistant that is able to: recover the nutritional information directly from a specific recipe, reason over recipes and diets with flexibility, i.e. by allowing some forms of diet disobedience, and persuade the user to minimize such acts of disobedience (Anselma et al., 2017).

The MADiMan architecture is composed by various modules (Fig. 1): a mobile app called CheckYourMeal!, a numerical reasoner that decides the compatibility of a specific dish in some point of the diet

\footnotetext{
${ }^{1} \mathrm{http} / / /$ di.unito.it/madiman
}

(Anselma et al., 2017), an information extraction module used to compute the nutrient values of a specific recipe, a Natural Language Generation service that converts the results of the computation to a textual form (Anselma and Mazzei, 2017).

In Fig. 1 we depict the architecture of the system implementing the MADiMan virtual dietitian. The information flow is: (1) A user, by using the CheckYourMeal! app, recovers the specific recipe of a dish which she wants to eat. (2) The app, communicating with the DietManager service, retrieves the user diet together with the list of the food that the user has eaten in the last days. (3) The NLU/IE module computes the salient nutrition information about the specific meal. (4) The Reasoner, using the user diet and the list of the food that she has been eaten in the last days, produces the final recommendation about the dish for the user. (5) The NLGenerator uses the recommendation given by the Reasoner, produces an explanation for the user in simple natural language. (6) The DietManager sends the result produced by the NLGenerator to the app: the user will see this final result on her smartphone. If the user decides to eat the dish, the app will send this information to the DietManager that will update the list of food eaten.

A crucial point in the proposal of new methods for helping people with computer is their evaluation. With this aim, some different paradigms have been used for evaluating MADiMan.

At first, a simulation was performed by using virtual agents, modeling different kinds of users, and real data regarding hospital menus, for evaluating the nu- 
meric reasoning module of MADiMan. In particular, it has been proved that MADiMan overcomes a reasonable baseline (Anselma et al., 2017). Then, the same experiment has been replicated by using menus from the Gedeone database, that is a collection of 500 Mediterranean recipes annotated with their macronutrient contents (Anselma et al., 2018).

More recently, it has been developed the CheckYourMeal! app in order to evaluate the performance and the usability of the whole architecture with human evaluation into a realistic context. This app has been used in a first human-evaluation experiment to test the appealing of the automatically generated text messages (Anselma and Mazzei, 2018). The authors asked the testers to play a diet game for a simulated period of two weeks, spending at least 15 minutes of their time. A tester had to imagine to eat for two weeks in a restaurant: for each slot of the week (breakfast, lunch, supper from Monday to Sunday) she had to choose only among the menus proposed in the app. In the simulation, the menus were randomly generated by considering the recipes of the Gedeone database (Anselma et al., 2018). During the game, the behaviour of the tester has been logged and at the end of the week a diet score has been automatically computed. This score measured the proximity on macronutrients to their ideal values. The logs of the interaction of the tester with MADiMAn contain information on which menus - and thus which dishes of the Gedeone recipe book - have been actually eaten by the user during the week ${ }^{2}$ (Anselma and Mazzei, 2018).

In this paper, we will use the information contained in the logs of the experiment described in (Anselma and Mazzei, 2018) as input data for computing the adherence of a tester to the Mediterranean diet. Indeed, the Mediterranean diet is often described as a number of prescriptions over one week.

In Section 3 we formalize the Mediterranean score by using ontological models and in Section 4 we use data from (Anselma and Mazzei, 2018) in order to validate the formalization.

\section{FORMALIZING THE MEDITERRANEAN SCORE BY USING ONTOLOGIES}

The Mediterranean diet has been largely investigated in the medical and cultural heritage literature since it has a proven relation with longevity and a healthy

\footnotetext{
${ }^{2}$ Moreover, at the end of the experiment, the testers had to compile a feedback web form.
}

life (Keys and Keys, 1975; Sofi et al., 2008; Estruch et al., 2013; Moro, 2016). In particular, the Mediterranean diet is an overall dietary pattern that has long been associated with lower incidence of cardiovascular disease and cancer (Keys et al., 1986). This diet is an example of a lifestyle guide that can be formalized as a set of qualitative constraints about various categories of foods and their frequency of consumption. Its formalization and application in a diet management system is particularly interesting since it can be viewed as a different source of information with respect to the standard account of calories among the various (macro and micro) nutrients. Moreover, as stated in (Panagiotakos et al., 2006): "It has been suggested that overall dietary patterns and not single nutrients should be studied, since food items might have a synergistic and antagonistic effect on health.".

In (Panagiotakos et al., 2006; Panagiotakos et al., 2007) the authors proposed a number of Mediterranean food intake reference values (MRVs) for computing a monthly Mediterranean diet score (MED). In Table 1 we adapt the MRVs reported in (Panagiotakos et al., 2006) after changing the frequency of consumption from month to week (e.g. a frequency of 5-8 servings/month becomes 1-2 servings/week). In the table there are eleven food categories and for each category a score in the range $0-5$ is given by considering the number of servings with respect to the MRV (so the MED score is an integer between 0 and 55). Most categories - i.e. those that include foods close to the Mediterranean dietary pattern - have a more is better score: for instance the Fruits category gives 5 points if the user assumes more than 4.5 portions in a week. In contrast, red meat, poultry, full fat dairy products - that are discouraged in the Mediterranean diet - have a less is better score: for instance the red meat category gives 5 points if the user does not eat any red meat at all during the week. An peculiar case is the consumption of alcoholic beverages, where a non-monotonic function has been adopted: the lowest score is given both when more than $700 \mathrm{ml} /$ day or $0 \mathrm{ml} /$ day is consumed (the motivation is that in the Mediterranean diet a moderate consumption of alcoholic beverages is recommended).

In order to compute the MED score, it is necessary to map the specific ingredients of a specific recipe into the categories of Table 1. In particular, for the experiments described in Section 2, we need to map the ingredients used for the Gedeone recipes. We decided to employ an ontology for this specific task and cast this problem as a typical ontological problem of instance checking. By starting from an existing ontology called PerkApp ${ }^{3}$ (Bailoni et al., 2016; Dragoni

\footnotetext{
${ }^{3}$ https://horus-ai.fbk.eu/helis/
} 


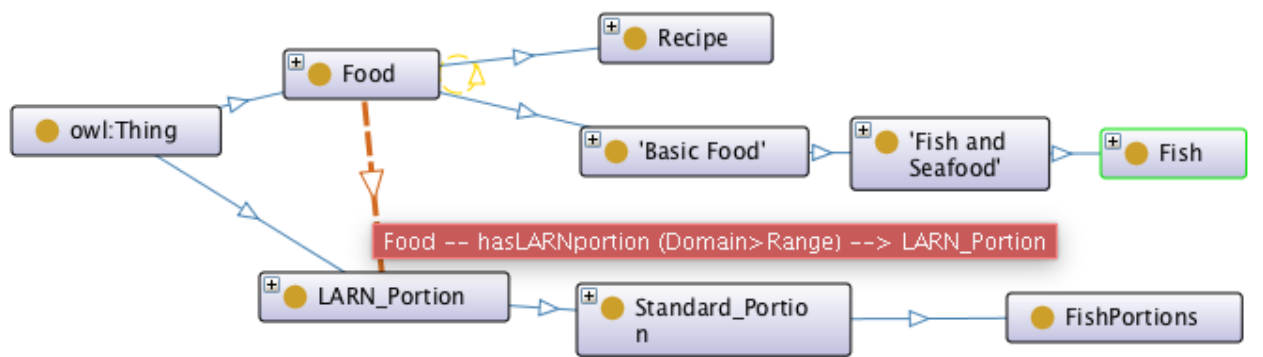

Figure 2: An Extended Branch of the PerkApp ontology showing the connection between Food types and LARN portions.

et al., 2017; Maimone et al., 2018; Donadello et al., 2019), we defined a number of subclasses needed to encode the Gedeone ingredients as instances of the ontology. The PerkApp ontology is built around the basic concepts of Food (e.g. Pasta alla Carbonara), Nutrient (e.g. Carbs, Protein, Lipids, Vitamin, Mineral), Timespan and Meal (e.g., Lunch, Afternoon). All these classes, and a number of derived subclasses, are necessary to encode the elements of a (healthy) diet.

We have extended the PerkApp ontology by including a class LarnPortion encoding a conversion table defined in (Società Italiana di Nutrizione Umana (SINU), 2014) that maps, for each Food type available in the ontology, the corresponding quantity in standard servings assumed in the Mediterranean Diet (more detailed information about the LARN are available in the next section). Fig. 2 presents a branch of the extended ontology connecting Food types and LARNPortion. Such expansion allowed us to model, for example, the standard Mediterranean Diet portion for the Food type Fish by explicitly including the axiom that any instance $x \in$ Fish has a corresponding FishPortion (a reified instance of the class LARN portion).

Such reified instance is then additionally linked, via the datatype property hasLARNvalue, to a corresponding integer representing the effective LARN value, in grams, for all the instances of the Fish category (that is 150 grams in such case). The same modelling procedure has been followed for each Food type available in the ontology. Such ontological extension, as a first consequence, directly helped us to deal with point (ii) described in the introduction (e.g., eggplants are vegetables) concerning the completion, with taxonomical information, about single instances of Food. In addition, such encoding allowed us to query the extended ontology in order to ask, for each instance of food acquired from the log coming from the CheckYourMeal! app, the associated portions assumed in the Mediterranean Diet, as indicated in the LARN guidelines (Società Italiana di Nutrizione Umana (SINU), 2014). Note that the mapping between the instances of food available in the $\log$ and the instances in the ontology is currently obtained with a simple stringmatching procedure. An example of SPARQL query extracting the corresponding Food category for an instance labelled as Swordfish, and additionally asking for the associated LARN portion is the following:

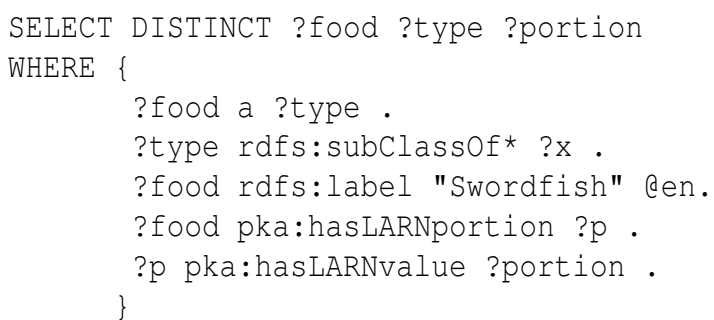

We have additionally started the population of the ontology with instances of Food belonging to the Gedeone ingredients that were not originally available in the PerkApp ontology. The current version of the ontology contains 150 out of 700 instances of the Gedeone ingredients.

\section{COMPUTING MED FROM REAL DATA}

The process used for the computation of the MED score starting from the simulation log produced in the experiment with CheckYourMeal! (Anselma and Mazzei, 2018) (see Section 2) can be understood as composed by four basic steps. Assuming to have the list and quantity of the food eaten during a week thanks to the respective recipes, we have to:

1. Convert the quantity of ingredients into grams,

2. Convert the quantity of ingredients in grams into standard servings defined in (Società Italiana di Nutrizione Umana (SINU), 2014),

3. Convert the ingredients into the corresponding MED category defined in (Panagiotakos et al., 2006) by using the food ontology described in Section 3, 
Table 1: The Mediterranean food intake reference values (MRVs) which are used to compute the MED diet score (adapted from (Panagiotakos et al., 2007; Panagiotakos et al., 2006)).

\begin{tabular}{|l|c|c|c|c|c|c|}
\hline & \multicolumn{5}{|c|}{ Frequency of consumption } \\
\hline How often do you consume (servings/week) & Never & $\mathbf{0 - 1}$ & $\mathbf{1 - 2}$ & $\mathbf{2 - 3}$ & $\mathbf{3 - 4 . 5}$ & $>\mathbf{4 . 5}$ \\
Non-refined cereals (whole grain bread, pasta, rice, etc.) & 0 & 1 & 2 & 3 & 4 & 5 \\
Potatoes & 0 & 1 & 2 & 3 & 4 & 5 \\
Fruits & 0 & 1 & 2 & 3 & 4 & 5 \\
Vegetables & 0 & 1 & 2 & 3 & 4 & 5 \\
Legumes & 0 & 1 & 2 & 3 & 4 & 5 \\
Fish & 0 & 1 & 2 & 3 & 4 & 5 \\
Red meat and products & 5 & 4 & 3 & 2 & 1 & 0 \\
Poultry & 5 & 4 & 3 & 2 & 1 & 0 \\
Full fat dairy products (cheese, yogurt, and milk) & 5 & 4 & 3 & 2 & 1 & 0 \\
\hline Use of olive oil in cooking (times/week) & Never & Rare & $\mathbf{0 - 1}$ & $\mathbf{1 - 3}$ & $\mathbf{3 - 5}$ & Daily \\
\hline Alcoholic beverages (ml/day, 100 ml=12 g ethanol) & $<\mathbf{3 0 0}$ & $\mathbf{3 0 0}$ & $\mathbf{4 0 0}$ & $\mathbf{5 0 0}$ & $\mathbf{6 0 0}$ & $>\mathbf{7 0 0}$ or 0 \\
\hline
\end{tabular}

Table 2: The weekly MED scores computed for three distinct users participating to the CheckYourMeal! experiment. The maximum score is 55 .

\begin{tabular}{|l|c|c|c|}
\hline & User 1 & User 2 & User 3 \\
\hline Non-refined cereals & 5 & 5 & 5 \\
Potatoes & 2 & 2 & 2 \\
Fruits & 3 & 5 & 4 \\
Vegetables & 5 & 5 & 5 \\
Legumes & 3 & 1 & 2 \\
Fish & 2 & 2 & 3 \\
Red meat and products & 4 & 5 & 4 \\
Poultry & 5 & 5 & 5 \\
Full fat dairy products & 1 & 1 & 3 \\
Use of olive oil & 5 & 5 & 5 \\
Alcoholic beverages & 5 & 5 & 5 \\
\hline MED score & 40 & 41 & 43 \\
& $(73 \%)$ & $(75 \%)$ & $(78 \%)$ \\
\hline
\end{tabular}

4. Sum and normalize the score of the categories over the week by using the scoring of Table 1 .

There are two challenging issues arising from step (1) of this process, that are: (i) in order to normalize all quantities in grams, we have to know the specific weight of each liquid mentioned in the recipe, and (ii) often some quantities occur under vague expressions ("a glass of water", "a handful of almonds", etc.). These problems can be solved assuming standard quantities for the various expressions (e.g. a glass of water contains approximately 200 milliliters and weighs approximately 200 grams) since conversions between expressions and volumes are quite standardized $^{4}$.

A key point of step (2) regards the encoding of

\footnotetext{
${ }^{4}$ See http://www.onlineconversion.com/weight_volume cooking.htm for a possible reference.
}

standard quantities for servings. We decided to use the standard quantities suggested by SINU (Società Italiana di Nutrizione Umana - Italian Society for $\mathrm{Hu}$ man Nutrition) (Società Italiana di Nutrizione Umana (SINU), 2014). SINU publishes the "LARN" (Livelli di Assunzione di Riferimento di Nutrienti ed energia per la popolazione italiana - Nutrient Reference Intake and Energy Levels for the Italian Population) (Società Italiana di Nutrizione Umana (SINU), 2014), which are the standard reference for the nutrition of Italian people (analogous documents exist for other countries); in particular in LARN it is possible to find a quantitative standard for defining a serving amount. For example, a serving of dried legumes is defined as 50 grams, corresponding to 3-4 tablespoons, and a serving of fresh legumes is defined as 150 grams, corresponding to half a plate. Note that the food categories described in LARN (Società Italiana di Nutrizione Umana (SINU), 2014) are distinct from the MED categories described in Table 1. Thus, we need to enrich the ontological model, as described in Section 3, with a number of subclasses connecting the two knowledge sources. For instance, we need to implement the concept of dried legume, which was not originally present in the PerkApp ontology.

In order to give an example of computation of the MED score, let us suppose that we found from the $\log$ that during the week the user ate 3 cups of whole grain rice. In step (1), knowing that a cup of whole grain rice is equivalent to 190 grams, this quantity is converted into 570 grams of whole-grain rice. In step (2), we find from LARN that a serving of whole grain rice is 80 grams, so that the user ate 7 servings of whole grain rice. In step (3), using ontological reasoning (i.e., SPARQL queries) we find that 7 servings 
Table 3: Amounts of macronutrients in kcal for the food ingested by the three users participating to the CheckYourMeal! experiment.

\begin{tabular}{|l|r|r|r|}
\hline & User 1 & User 2 & User 3 \\
\hline Carbohydrates taken & 9993 & 6810 & 15128 \\
Proteins taken & 1819 & 1200 & 1937 \\
Lipids taken & 4906 & 3845 & 7078 \\
\hline Total energy taken & 16718 & 11855 & 24143 \\
\hline
\end{tabular}

of whole grain rice correspond to 7 servings of nonrefined cereals. Finally, in step (4), from Table 1 we find that this quantity contributes to the weekly MED score with 5 points.

In Table 2 we report the MED scores for three distinct users participating to the CheckYourMeal! experiment. The three users have assumed considerably different amounts of food and of macronutrients, as shown by Table 3, where User 3 has consumed a high amount of food during the week (in average 3442 $\mathrm{kcal} /$ day), User 2 a low amount (1694 kcal/day) and User 1 an intermediate value $(2388 \mathrm{kcal} / \mathrm{day})$. The MED scores for the three users are similar (Table 2). One can notice that the use of a Mediterranean recipe book has resulted in a high MED score for all three users. In particular, there are high scores for the consumption of non-refined cereals, olive oil and vegetables for all the users, and a moderate score for the consumption of potatoes. This example confirms the intuition that quantitative constraints arising from dietary reference values on macronutrients and qualitative constraints arising from the principles of Mediterranean diet are two different notions that needed to be treated with different mechanisms.

\section{CONCLUSIONS}

In this paper we have described a first proposal for the ontological formalization of the Mediterranean score MED as proposed in (Panagiotakos et al., 2007; Panagiotakos et al., 2006) as an extension of the food ontology PerkApp (Maimone et al., 2018). Moreover, we have discussed how to effectively implement the computation of MED in the framework of MADiMan, a system for diet management. By using data from the CheckYourMeal! experiment, we have computed in MADiMan the MED score for three distinct users and so we have empirically showed that macronutrients and MED score are independent measures for rating the quality of meals for a healthy diet, and as a consequence it necessary to compute both independently.

To the best of our knowledge, this is the first work that formalizes the MED score in terms of ontolog- ical constraints and proposes an effective procedure for computing it.

In future work we intend to use the computation of the MED score to augment the answers returned by MADiMan with an explanation about the compatibility of a specific dish with the Mediterranean diet.

\section{REFERENCES}

Anselma, L. and Mazzei, A. (2015). Towards Diet Management with Automatic Reasoning and Persuasive Natural Language Generation. In Progress in Artificial Intelligence - 17th Portuguese Conference on Artificial Intelligence, EPIA 2015, Coimbra, Portugal, September 8-11, 2015. Proceedings, pages 79-90.

Anselma, L. and Mazzei, A. (2017). An approach for explaining reasoning on the diet domain. In Basile, P., Croce, D., and Guerini, M., editors, Proc. of the 1st Workshop on Natural Language for Artificial Intelligence co-located with AI*IA 2017, Bari, Italy., volume 1983 of CEUR Workshop Proceedings, pages 4 17. CEUR-WS.org.

Anselma, L. and Mazzei, A. (2018). Designing and testing the messages produced by a virtual dietitian. In Krahmer, E., Gatt, A., and Goudbeek, M., editors, Proceedings of the 11th International Conference on Natural Language Generation, Tilburg University, The Netherlands, November 5-8, 2018, pages 244-253. Association for Computational Linguistics.

Anselma, L., Mazzei, A., and De Michieli, F. (2017). An artificial intelligence framework for compensating transgressions and its application to diet management. Journal of Biomedical Informatics, 68:58-70.

Anselma, L., Mazzei, A., and Pirone, A. (2018). Automatic reasoning evaluation in diet management based on an Italian cookbook. In Proceedings of the Joint Workshop on Multimedia for Cooking and Eating Activities and Multimedia Assisted Dietary Management, CEA/MADiMa '18, pages 59-62, New York, NY, USA. $\mathrm{ACM}$.

Bailoni, T., Dragoni, M., Eccher, C., Guerini, M., and Maimone, R. (2016). Healthy lifestyle support: the perkapp ontology. In OWL: Experiences and Directions-Reasoner Evaluation, pages 15-23. Springer.

Bas, E. (2014). A robust optimization approach to diet problem with overall glycemic load as objective function. Applied Mathematical Modelling, 38(19-20):4926 4940.

Buisson, J.-C. (2008). Nutri-educ, a nutrition software application for balancing meals, using fuzzy arithmetic and heuristic search algorithms. Artif. Intell. Med., 42(3):213-227.

Donadello, I., Dragoni, M., and Eccher, C. (2019). Persuasive explanation of reasoning inferences on dietary data. In Demidova, E., Dietze, S., Breslin, J. G., Gottschalk, S., Cimiano, P., Ell, B., Lawrynowicz, A., Moss, L., and Ngomo, A. N., editors, Joint Proceedings of the 6th International Workshop on Dataset 
PROFlLing and Search \& the 1st Workshop on Semantic Explainability co-located with the 18th International Semantic Web Conference (ISWC 2019), Auckland, New Zealand, October 27, 2019, volume 2465 of CEUR Workshop Proceedings, pages 46-61. CEUR-WS.org.

Dragoni, M., Bailoni, T., Eccher, C., Guerini, M., and Maimone, R. (2017). A semantic-enabled platform for supporting healthy lifestyles. In Proceedings of the Symposium on Applied Computing, SAC '17, pages 315-322, New York, NY, USA. ACM.

Estruch, R., Ros, E., Salas-Salvadó, J., Covas, M.-I., Corella, D., Arós, F., Gómez-Gracia, E., RuizGutiérrez, V., Fiol, M., Lapetra, J., et al. (2013). Primary prevention of cardiovascular disease with a mediterranean diet. New England Journal of Medicine, 368(14):1279-1290.

Keys, A. and Keys, M. (1975). How to Eat Well and Stay Well the Mediterranean Way. Doubleday.

Keys, A., Mienotti, A., Karvonen, M. J., Aravanis, C., Blackburn, H., Buzina, R., Djordjevic, B., Dontas, A., Fidanza, F., Keys, M. H., et al. (1986). The diet and 15 -year death rate in the seven countries study. American journal of epidemiology, 124(6):903-915.

Lancaster, L. M. (1992). The history of the application of mathematical programming to menu planning. European Journal of Operational Research, 57(3):339 347.

Maimone, R., Guerini, M., Dragoni, M., Bailoni, T., and Eccher, C. (2018). Perkapp: A general purpose persuasion architecture for healthy lifestyles. Journal of Biomedical Informatics, 82:70-87.

Mazzei, A. (2014). On the lexical coverage of some resources on Italian cooking recipes. In Proc. of CLiC-it 2014, First Italian Conference on Computational Linguistics, pages 254-259.

Mazzei, A., Anselma, L., De Michieli, F., Bolioli, A., Casu, M., Gerbrandy, J., and Lunardi, I. (2015). Mobile computing and artificial intelligence for diet management. Lecture Notes in Computer Science, 9281:342349.

Moro, E. (2016). The Mediterranean diet from Ancel Keys to the UNESCO cultural heritage. A pattern of sustainable development between myth and reality. Procedia - Social and Behavioral Sciences, 223:655 - 661. 2nd International Symposium NEW METROPOLITAN PERSPECTIVES - Strategic planning, spatial planning, economic programs and decision support tools, through the implementation of Horizon/Europe2020. ISTH2020, Reggio Calabria (Italy), 18-20 May 2016.

Panagiotakos, D. B., Pitsavos, C., Arvaniti, F., and Stefanadis, C. (2007). Adherence to the Mediterranean food pattern predicts the prevalence of hypertension, hypercholesterolemia, diabetes and obesity, among healthy adults; the accuracy of the meddietscore. Preventive medicine, 44(4):335-340.

Panagiotakos, D. B., Pitsavos, C., and Stefanadis, C. (2006). Dietary patterns: a Mediterranean diet score and its relation to clinical and biological markers of cardiovascular disease risk. Nutrition, Metabolism and Cardiovascular Diseases, 16(8):559-568.
Società Italiana di Nutrizione Umana (SINU) (2014). LARN: Livelli di Assunzione di Riferimento di $\mathrm{Nu}$ trienti ed Energia per la Popolazione Italiana.

Sofi, F., Cesari, F., Abbate, R., Gensini, G. F., and Casini, A. (2008). Adherence to mediterranean diet and health status: meta-analysis. Bmj, 337:a1344. 